\title{
Estimulación multipunto mediante captura anódica del ventrículo izquierdo a través de un electrodo cuadripolar: evaluación hemodinámica no invasiva
}

\section{Jorge Toquero Ramos ${ }^{a, *}$, Manuel Sánchez García ${ }^{a}$, Lorena Ruíz Bautista ${ }^{a}$, Víctor Castro Urda ${ }^{a}$, Sara Alyoun Alonso ${ }^{b}$ e Ignacio Fernández Lozano ${ }^{a}$}

\author{
a Servicio de Cardiología, Hospital Universitario Puerta de Hierro Majadahonda, Madrid, España \\ b St Jude Medical, España
}

Recibido el 9 de marzo de 2015; aceptado el 16 de julio de 2015

Disponible en Internet el 1 de octubre de 2015

\author{
PALABRAS CLAVE \\ Dispositivo; \\ Estimulación \\ biventricular; \\ Hemodinamia; \\ In vivo
}

\begin{abstract}
Resumen
Introducción: Un elevado porcentaje de pacientes no responde a la resincronización. La captura anódica izquierda mediante estimulación a alto voltaje, permite una estimulación multipunto que podría aumentar la tasa de respondedores.

Objetivo: Evaluar la viabilidad y eficacia hemodinámica aguda de estimulación a alto voltaje mediante el uso del electrodo cuadripolar Quartet $1458 Q^{\circledR}$.

Métodos: Se incluyeron 18 pacientes consecutivos, con electrodo cuadripolar, en quienes se confirmó captura en el modo deseado mediante monitorización electrocardiográfica. Se compararon las diferencias hemodinámicas agudas de captura anódica (electrodos 1-cátodo- y 4-ánodo, máximo voltaje y anchura), biventricular y ventricular derecha. Mediante el Task Force Monitor, se hizo análisis hemodinámico agudo de presión arterial sistólica, diastólica y media, volumen latido e índice, gasto cardiaco e índice. Para cada variable se analizó el valor máximo, mínimo y promedio, durante fases estables de diez minutos.

Resultados: Se analizaron 18 pacientes (5 mujeres-27,8\%), con edad media de 67,2 años (37-81); $33 \%$ con fibrilación auricular permanente y $39 \%$ con cardiopatía isquémica (sólo 5,6\% ambas). La estimulación anódica aumentó significativamente el volumen latido e índice, así como el gasto cardiaco e índice en comparación con el modo biventricular [76,21 vs. 71,04 ml ( $p=0,014) ; 40,67$ vs. $37,88 \mathrm{ml} / \mathrm{m}^{2}$ ( $\left.p=0,018\right) ; 5,29$ vs. $4,89 \mathrm{l} / \mathrm{min}(\mathrm{p}=0,007) ; 2,86$ vs. $2,65 \mathrm{l} / \mathrm{min} / \mathrm{m}^{2}$ ( $\left.p=0,007\right)$, respectivamente]. En el análisis por subgrupos, se concentró el beneficio en los pacientes en ritmo sinusal.
\end{abstract}

Abreviaturas: EMP, Estimulación multipunto; PA, Presión arterial (S-sistólica, D-diastólica y M-media); VL, Volumen latido; iVL, Índice del volumen latido; GC, Gasto cardiaco; IC, Índice cardiaco.

* Autor para correspondencia.

Correo electrónico: jorgetoquero@secardiologia.es (J. Toquero Ramos). 
Conclusión: La estimulación multipunto mediante captura anódica a través de un electrodo cuadripolar es factible, demostrándose así diferencias significativas en el volumen latido y gasto cardiaco, aunque limitado a la población en ritmo sinusal.

(c) 2015 Sociedad Colombiana de Cardiología y Cirugía Cardiovascular. Publicado por Elsevier España, S.L.U. Este es un artículo Open Access bajo la CC BY-NC-ND licencia (http:// creativecommons.org/licencias/by-nc-nd/4.0/).

\section{KEYWORDS}

Device;

Biventricular

stimulation;

Hemodynamics;

In vivo

\section{Multipoint stimulation by means of anodal capture of left ventricle using a quadripolar lead: non-invasive hemodynamic evaluation}

\begin{abstract}
Introduction: A high percentage of patients does not respond to resynchronization. Left anodal capture by means of high-voltage stimulation allows a multipoint stimulation that could increase the responders rate.

Objetive: To assess viability and acute hemodynamic effectiveness of high voltage stimulation using the quadripolar lead Quartet $1458 Q^{\circledR}$.

Methods: 18 consecutive patients with quadripolar lead were included. The capture was confirmed by means of electrocardiographic monitoring. Acute hemodynamic differences of anodal capture were compared (lead 1-cathode and 4-anode, maximum voltage and width), biventricular and right ventricular. Using the Task Force Monitor an acute hemodynamic analysis of systolic, diastolic and average blood pressure, stroke volume and index, cardiac output and index was conducted. For each variable the maximum, minimum and average values were reached during ten minute stable phases.

Results: 18 patients (5 women - 27.8\%) were analyzed, with an average age of 67.2 years (3781); $33 \%$ with permanent atrial fibrillation and $39 \%$ with ischemic cardiopathology (only $5.6 \%$ with both). Anodal stimulation significantly raised the stroke volume and index, as well as the cardiac output and index compared to the biventricular mode [76.21 vs. $71.04 \mathrm{ml}(p=0.014)$; 40.67 vs. $37.88 \mathrm{ml} / \mathrm{m}^{2}(p=0.018) ; 5.29$ vs. $4.89 \mathrm{l} / \mathrm{min}(p=0.007) ; 2.86$ vs. $2.65 \mathrm{l} / \mathrm{min} / \mathrm{m}^{2}$ $(p=0.007)$, respectively]. Subgroup analysis focused on the benefit for patients in sinus rhythm. Conclusion: Multipoint stimulation by means of anodal capture using a quadripolar lead is possible. This further proves significant improvement in the stroke volume and cardiac output, though limited to the population in sinus rhythm.

(c) 2015 Sociedad Colombiana de Cardiología y Cirugía Cardiovascular. Published by Elsevier España, S.L.U. This is an open access article under the CC BY-NC-ND license (http:// creativecommons.org/licenses/by-nc-nd/4.0/).
\end{abstract}

\section{Introducción}

La terapia de resincronización cardiaca (TRC) ha demostrado ampliamente en los últimos años en gran variedad de estudios, su eficacia en el tratamiento de pacientes con insuficiencia cardiaca avanzada, disfunción ventricular severa y trastornos de la conducción ${ }^{1-3}$. No obstante, hasta un $40 \%$ de pacientes sometidos a esta terapia presentan escasa o nula respuesta al efecto clínico y hemodinámico producido por la misma a largo plazo (seis meses), siendo catalogados como «no respondedores» 4,5 . Esto ha obligado a recalcar continuamente la necesidad de optimizar la programación de estos dispositivos, ajustando los intervalos AV y VV con base en parámetros electrocardiográficos, ecocardiográficos o hemodinámicos ${ }^{6,7}$. Sin embargo, a pesar de las recomendaciones de optimización de todos los pacientes portadores de un dispositivo de TRC, pocos centros implantadores la realizan, no solo por el costo y tiempo que conlleva sino por el entrenamiento adicional, ocasionalmente complejo, que requiere ${ }^{8}$.

Una alternativa propuesta recientemente es la optimización de la TRC con base en la determinación del gasto cardiaco de forma no invasiva a través de distintos sistemas de medición, lo que supondría un método más seguro, económico, y sobre todo más objetivo en comparación con otros como la ecocardiografía ${ }^{9,10}$. La medición del gasto cardiaco y otras variables relacionadas, proporciona información pronóstica independiente y sólida en pacientes con insuficiencia cardiaca ${ }^{11}$, y se ha demostrado recientemente que la optimización de la TRC basada en estos parámetros hemodinámicos no invasivos, se asocia con incremento del remodelado reverso del ventrículo izquierdo (VI), mejoría en la fracción de eyección y mayor respuesta clínica ${ }^{12}$. Por otra parte, el avance tecnológico de la TRC ha permitido que hoy en día la estimulación ventricular izquierda pueda realizarse en diferentes configuraciones de polaridad del electrodo implantado en el ventrículo izquierdo o entre este y el implantado en el ventrículo derecho (VD), con el objetivo de garantizar la captura ventricular con el menor voltaje y consumo de batería, así como evitar estimulación frénica indeseable: bipolar, monopolar (en la cual el cátodo lo constituye el electrodo de la punta del cable y el ánodo la carcasa del generador) o pseudobipolar (el ánodo del cable situado en el VD es común para ambos ventrículos). 
Diferentes trabajos en la literatura en los últimos años, han evaluado el potencial de la captura anódica en los pacientes portadores de TRC. La captura anódica se produce cuando alrededor del ánodo (polo positivo), al estimular a voltajes elevados, se produce una ordenación de cargas negativas que pueden ser capaces de generar una despolarización miocárdica a partir de dicho punto. En otras palabras, al incrementar la intensidad de estimulación del músculo cardiaco, el potencial de acción se origina de «electrodos virtuales» de mayor tamaño que el tamaño físico del cátodo que inicia el estímulo. Los pulsos anódicos hiperpolarizan el tejido y pueden producir una activación durante el pulso o al finalizar el mismo (estimulación de corrientes de entrada activadas por hiperpolarización que despolarizan la membrana hasta el umbral del potencial de acción al final del pulso). Este efecto, conocido desde hace años especialmente en los desfibriladores con electrodo bipolar integrado de VD, que condicionaba más frecuentemente captura anódica dada la mayor superficie del mismo, ha sido recientemente evaluado en pacientes portadores de $\mathrm{TRC}^{13}$.

De cara a la resincronización, la estimulación anódica tiene la ventaja potencial de generar dos cátodos, uno real y uno «virtual» sobre el miocardio, produciendo, en teoría, una despolarización miocárdica más rápida y coordinada al «lanzar» frentes de onda desde localizaciones más distales a la posición física del electrodo. Si bien tradicionalmente ésta se ha considerado como perjudicial, al requerir mayores umbrales para la captura miocárdica o poder interferir en la programación adecuada del intervalo $\mathrm{VV}$ respecto a la estimulación catódica, la captura anódica puede en ocasiones tener un efecto beneficioso, ya que da lugar a una estimulación simultánea multipunto (VI, VD proximal o ánodo y VD distal o cátodo), lo que genera un patrón de resincronización óptimo, que puede objetivarse por parámetros electrocardiográficos (anchura QRS), ecocardiográficos (Doppler tisular) y hemodinámicos (gasto cardiaco) ${ }^{14}$.

La introducción en el mercado de un nuevo electrodo cuadripolar para la estimulación ventricular izquierda (St. Jude Medical Quartet $1458 Q^{\circledR}$ ), con múltiples vectores de programación posibles entre los cuatro electrodos del VI y sus respectivas combinaciones con los del VD, ha llevado a plantear la posibilidad de lograr captura anódica en el electrodo del VI, obteniendo de esa manera estimulación «multipunto» en dicho ventrículo, que podría asociarse a la optimización de parámetros hemodinámicos agudos $\mathrm{y}, \mathrm{a}$ priori, a la mejoría pronóstica a mediano y largo plazo.

El objetivo de este estudio es valorar la eficacia de la captura anódica del VI mediante empleo de electrodo cuadripolar SJM Quartet $1458 Q^{\circledR}$ en pacientes portadores de un dispositivo de TRC mediante la determinación de parámetros hemodinámicos agudos de forma no invasiva, tales como la presión arterial sistólica, diastólica y media, el volumen latido e índice del volumen latido o el gasto e índice cardiacos.

\section{Métodos}

Para llevar a cabo este estudio se reclutaron 18 pacientes consecutivos en quienes se implantó un dispositivo de TRC con electrodo del VI cuadripolar y que aceptaron participar en el estudio. La decisión de implantar dicho electrodo se tomó con base en criterios clínicos y/o decisión médica previa. Dicho electrodo es especialmente beneficioso en pacientes con elevado umbral de estimulación del ventrículo izquierdo o estimulación frénica difícilmente controlable; los múltiples vectores de estimulación programables permiten buscar el mejor umbral, reduciendo el consumo de batería del dispositivo a largo plazo, a fin de evitar estimulación frénica indeseable. En ningún caso la indicación de dicho electrodo estuvo condicionada por la participación en el presente estudio. El trabajo fue aprobado por el comité ético de investigación clínica del hospital.

El estudio se llevó a cabo en diferentes fases:

- Fase 1: Reclutamiento de pacientes. Se hizo contacto telefónico con los pacientes portadores de un dispositivo de TRC con un electrodo cuadripolar Quartet $1458 Q^{\circledR}$ en el VI (implantados en el año previo). En una primera visita se detallaron las características del proyecto y se solicitó la participación voluntaria en el mismo. Una vez obtenido el consentimiento informado del paciente se agendó una cita única para la programación del dispositivo de acuerdo con el protocolo y las mediciones.

- Fase 2: Programación del dispositivo y recolección de datos. Según las citas previamente acordadas con cada paciente, se procedió a la medición de los parámetros hemodinámicos descritos (presión arterial, volumen latido, índice del volumen latido, gasto e índice cardiacos) de forma no invasiva, mediante el Task Force Monitor. Todo el protocolo se realizó bajo monitorización electrocardiográfica continua. Se determinó de forma automática la duración del complejo QRS y se analizaron de manera minuciosa los cambios de morfología para garantizar las tres fases de estudio buscadas: estimulación multipunto mediante captura anódica del VI, estimulación biventricular verdadera y estimulación sólo del VD.

Se programó el dispositivo de TRC en las tres modalidades y se realizaron las mediciones hemodinámicas no invasivas correspondientes. Cada una de las programaciones se mantuvo durante un tiempo mínimo de $10 \mathrm{~min}$, de manera que permitiera la estabilización de parámetros hemodinámicos, redujera las variaciones asociadas al estrés y se registraran de forma continua las variables descritas para su análisis posterior. Dichas programaciones fueron:

- Estimulación a máximo voltaje y anchura de impulso $1,5 \mathrm{~ms}$ para asegurar captura anódica del VI (QRS generalmente de menor duración en captura anódica, frente a captura bipolar verdadera, con cambios morfológicos claros no atribuibles al artefacto generado por la espícula de estimulación a máximo voltaje).

- Estimulación bipolar verdadera del VI, manteniendo anchura de impulso en $1,5 \mathrm{~ms}$, con decremento progresivo del voltaje de estimulación, asegurando la captura biventricular por la morfología registrada en el electrocardiograma de superficie (anchura y eje del QRS).

- Estimulación sólo del VD, manteniendo anchura de impulso en $1,5 \mathrm{~ms}$, con decremento progresivo del voltaje de estimulación, asegurando pérdida de captura del VI por los cambios registrados en el electrocardiograma de superficie (anchura y eje del QRS).

Tras la realización de las mediciones, se configuró nuevamente el dispositivo de TRC según la programación inicial. 
Ningún parámetro fue modificado, salvo en caso de detectar, durante la realización del protocolo, alguna anomalía de funcionamiento en el dispositivo que requiriese su corrección.

- Todas las mediciones de parámetros hemodinámicos se llevaron a cabo mediante el empleo del Task Force Monitor. El sistema ha sido validado para el estudio del síncope, proporcionando, de forma no invasiva, información latido a latido de parámetros hemodinámicos y autonómicos. En la actualidad es el estándar recomendado por las Guías Europeas para el estudio de síncope. Para ello el sistema usa una combinación de electrocardiografía, cartografía de impedancia y presión arterial oscilométrica y continua. Se colocaron seis electrodos en el tórax de cada paciente, uno en la nuca y uno en la pierna, además de un manguito en el brazo para medir la presión arterial oscilométrica, un manguito en los dedos para la presión arterial continua, y un pulsioxímetro. Todos esos dispositivos de medición son coordinados e integrados por el software del Monitor para, mediante su análisis conjunto, aportar de forma numérica y gráfica los parámetros hemodinámicos no invasivos de interés.

- Fase 3: Análisis de los resultados. Se realizaron las siguientes mediciones: intervalo RR, frecuencia cardiaca (FC), presión arterial (sistólica -PAS-, diastólica -PAD-, media -PAM-) y presión del pulso (PP), tiempo de eyección del VI (LVET), período pre-eyección (PEP), volumen de expulsión cardiaco (VEC), índice de expulsión (IEC), volumen minuto cardiaco ( $\mathrm{VM} / \mathrm{CO})$, resistencia periférica total (RPT), contenido líquido torácico (TFC), e índice de contractilidad (IC). Igualmente se analizaron en detalle los valores de voltaje y anchura de pulso necesarios para garantizar la estimulación anódica.

Los resultados fueron analizados con el programa estadístico SPSS versión 15.0. Cada variable de interés se analizó teniendo en cuenta un factor intrasujetos (fase de estimulación, excluyendo la medición basal). A pesar del tamaño reducido de la muestra, las distribuciones de las puntuaciones siguieron aproximadamente la función de distribución normal, por lo que se realizaron pruebas paramétricas. Para cada medida y par de variables, se utilizó la prueba t de Student para medidas relacionadas. En caso de incumplimiento del supuesto de normalidad para pruebas paramétricas, se utilizó la prueba de Wilcoxon. Todos los análisis se realizaron considerando un nivel de significación a priori $(\alpha)$ de 0,05 . Sin embargo, al hacer tres comparaciones por indicador (anódica-biventricular, anódica-ventrículo derecho y biventricular-ventrículo derecho), se controló la tasa de error por familia de comparaciones mediante la corrección de Bonferroni, estableciendo un $\alpha \mathrm{F}=0,017$. El valor promedio es el mejor indicador de una variable para un sujeto en cada fase. Los valores mínimo y máximo suelen estar sesgados por valores atípicos debidos al azar muestral. No obstante, se presentan los resultados de las comparaciones para los valores máximo, mínimo y promedio.

\section{Resultados}

Se incluyeron un total de 18 pacientes consecutivos ( 5 mujeres; $27,8 \%$ ), con edades entre 37 y 81 años (media 67,2 años;
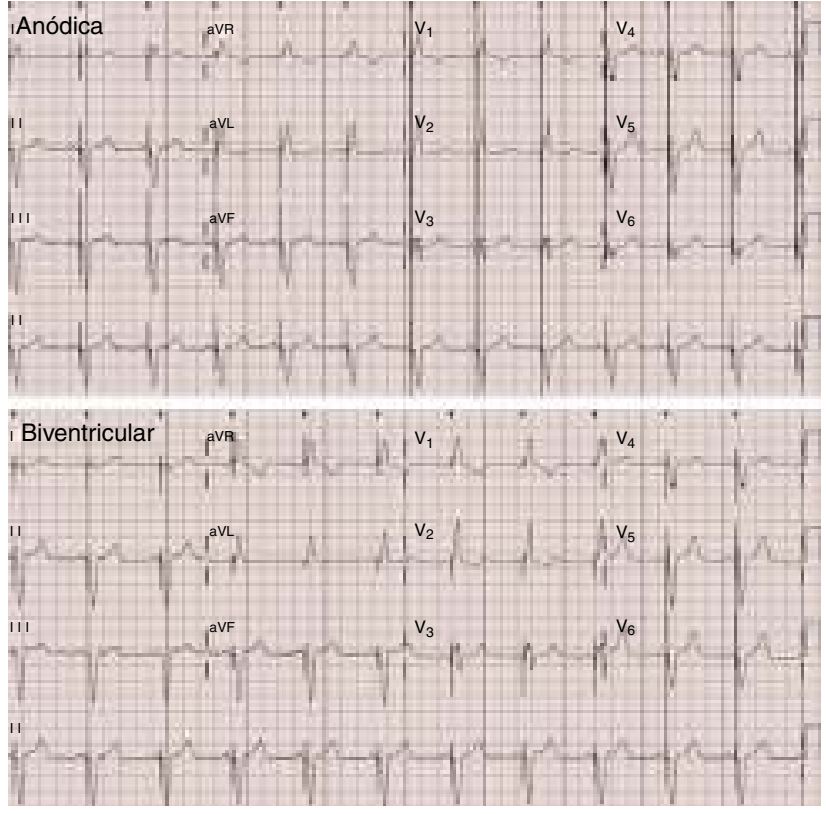

Figura 1 Comparación electrocardiográfica con estimulación anódica (arriba) frente a biventricular (abajo). Nótese el cambio en derivaciones como I y aVR.

DT: 10,2). Del total de la muestra, 6 pacientes (33\%) presentaban FA permanente y $7(38,8 \%)$ cardiopatía isquémica. Sólo uno $(5,6 \%)$ combinaba ambas patologías.

En todos los casos se documentó la captura anódica mediante cambios en el electrocardiograma de superficie (figs. 1 y 2). En este caso no se observaron diferencias significativas en la duración del complejo QRS al comparar la estimulación anódica frente a la estimulación biventricular convencional. Las mediciones obtenidas en situación

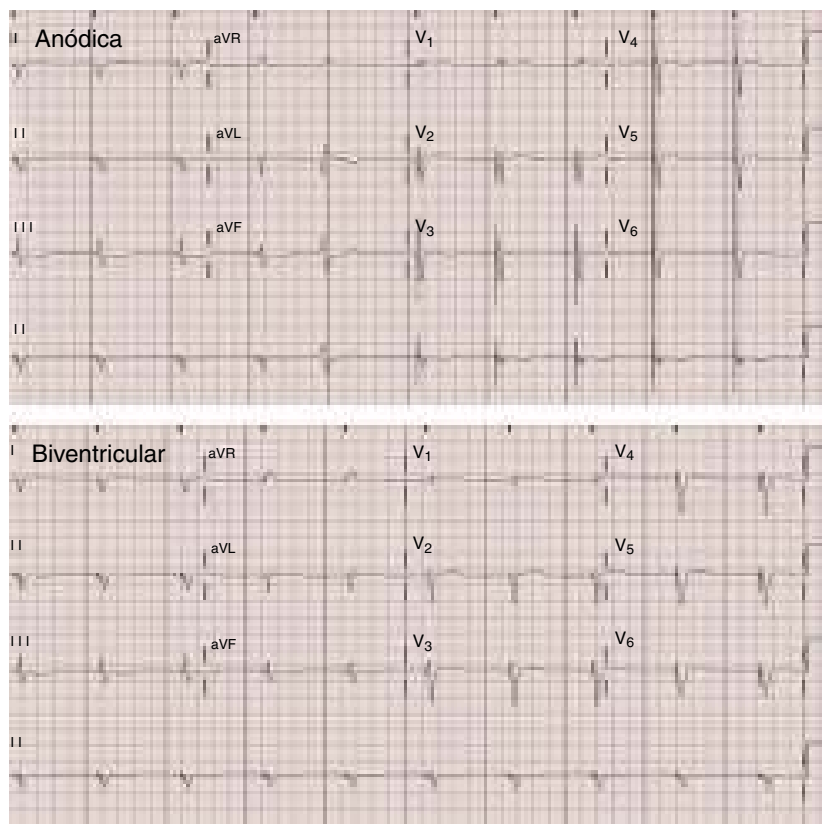

Figura 2 Comparación electrocardiográfica con estimulación anódica (QRS $110 \mathrm{~ms}$ ) frente a biventricular (QRS $129 \mathrm{~ms}$ ). Nótese el estrechamiento del complejo QRS. 
Tabla 1 Media (DT) de los valores máximos, mínimos y promedio de cada variable en las cuatro fases

\begin{tabular}{|c|c|c|c|c|}
\hline & Basal & EMP-anódica & $\mathrm{BiV}$ & VD \\
\hline \multicolumn{5}{|l|}{$F C$} \\
\hline Máx. & $98,47(46)$ & $92,42(34,7)$ & $90,93(26,8)$ & $94,27(28,8)$ \\
\hline Mín. & $57,24(8,8)$ & $58,99(7,1)$ & $56,82(11,5)$ & $58,06(8,6)$ \\
\hline Prom. & $70,52(8,4)$ & $70,36(9,1)$ & $69,99(8)$ & $69,93(7,7)$ \\
\hline \multicolumn{5}{|l|}{ PAS } \\
\hline Máx. & $128,04(20,6)$ & $136,21(30)$ & $134,46(19,5)$ & $135,98(29,7)$ \\
\hline Mín. & $100,38(16,2)$ & $94,73(16,1)$ & $99,24(16,3)$ & 101,65 (19) \\
\hline Prom. & $115,28(16,5)$ & $114,05(16,1)$ & $115,92(13,4)$ & 118,83 (19) \\
\hline \multicolumn{5}{|l|}{$P A D$} \\
\hline Máx. & $84,71(11,3)$ & $88,52(20,9)$ & $88,01(18,3)$ & $89,78(19,5)$ \\
\hline Mín. & $63,18(10,2)$ & $61,03(16,3)$ & $63,1(13)$ & $63,23(12,5)$ \\
\hline Prom. & $74,19(10,1)$ & $70,96(12)$ & $74,48(11,1)$ & $76,93(14,3)$ \\
\hline \multicolumn{5}{|l|}{ PAM } \\
\hline Máx. & $98,5(14,7)$ & $101,96(24,1)$ & $101,94(14,4)$ & $104,44(21,8)$ \\
\hline Mín. & 76,94 (14) & 76,42 (17) & $78,32(14,4)$ & 81,01 (17) \\
\hline Prom. & $88,92(12,8)$ & $85,25(15,4)$ & $89,92(12,1)$ & $92,28(16,7)$ \\
\hline \multicolumn{5}{|l|}{$V L$} \\
\hline Máx. & $91,83(52,4)$ & $97,13(50,3)$ & $95,1(56,2)$ & $89,87(41,3)$ \\
\hline Mín. & $55,46(36,1)$ & $63,58(43,6)$ & $59,15(40,9)$ & $53,49(28,8)$ \\
\hline Prom. & $69,33(38,2)$ & $76,21(44,7)$ & $71,04(43)$ & $69,63(33,8)$ \\
\hline \multicolumn{5}{|l|}{ IVl } \\
\hline Máx. & $49,26(27,5)$ & $52,04(26,9)$ & $52,96(30,2)$ & $48,26(22,2)$ \\
\hline Mín. & $29,89(20)$ & $34,14(23,2)$ & $31,81(22,1)$ & $28,96(16,3)$ \\
\hline Prom. & $37,19(20,8)$ & $40,67(23,3)$ & $37,88(23)$ & $37,47(18,5)$ \\
\hline \multicolumn{5}{|l|}{$G C$} \\
\hline Máx. & $7,49(2,7)$ & $8,37(4,6)$ & $7,4(3,6)$ & $6,99(2,6)$ \\
\hline Mín. & $3,49(2,4)$ & $4,11(2,9)$ & $3,64(2,4)$ & $3,33(1,7)$ \\
\hline Prom. & $4,85(2,5)$ & $5,29(2,9)$ & $4,89(2,9)$ & $4,91(2,2)$ \\
\hline \multicolumn{5}{|l|}{ IC } \\
\hline Máx. & $4,07(1,6)$ & $4,64(2,7)$ & $4,2(2,4)$ & $3,77(1,5)$ \\
\hline Mín. & $1,9(1,3)$ & $2,22(1,5)$ & $1,97(1,3)$ & $1,82(1,1)$ \\
\hline Prom. & $2,62(1,4)$ & $2,86(1,6)$ & $2,65(1,6)$ & $2,65(1,3)$ \\
\hline
\end{tabular}

FC: frecuencia cardiaca; PAS: presión arterial sistólica; PAD: presión arterial diastólica; PAM: presión arterial media; VL: volumen latido; iVL: índice volumen latido; GC: gasto cardiaco; IC: índice cardiaco. EMP-anódica: estimulación multipunto anódica; BiV: biventricular; VD: ventrículo derecho.

basal, con estimulación anódica (EMP-anódica), estimulación biventricular (BiV) y sólo del VD de las distintas variables se muestran en la tabla 1 . El análisis estadístico de las medias de las distintas variables en las tres fases se muestra en la tabla 2. En comparación con la estimulación BiV, la estimulación EMP-anódica aumentó significativamente el VL $(76,21$ vs. 71,$04 ; p=0,014)$, el $G C(5,29$ vs. 4,$91 ; p=0,007)$ y el IC (2,86 vs. 2,$65 ; p=0,007)$. Dicho beneficio no puede explicarse por un potencial deterioro hemodinámico de la estimulación VD o BiV, como muestra el incremento de

Tabla 2 Comparación estimulación anódica frente a estimulación BiV y de VD

\begin{tabular}{lcccc}
\hline & EMP-anódica & Estimulación BiV & Estimulación VD & P EMP - BiV \\
\hline FC $(\mathrm{lpm})$ & $70,36(9,1)$ & $69,99(8)$ & $69,93(7,7)$ & 0,323 \\
PAS $(\mathrm{mm} \mathrm{Hg})$ & $114,01(16,1)$ & $115,92(13,4)$ & $118,83(19)$ & 0,211 \\
PAD $(\mathrm{mm} \mathrm{Hg})$ & $70,96(12)$ & $74,48(11,1)$ & $76,93(14,3)$ & 0,045 \\
PAM $(\mathrm{mm} \mathrm{Hg})$ & $82,25(15,4)$ & $89,92(12,1)$ & $92,28(16,7)$ & 0,027 \\
VL $(\mathrm{ml})$ & $76,21(44,7)$ & $71,04(43)$ & $69,93(33,8)$ & 0,014 \\
iVL $\left(\mathrm{ml} / \mathrm{m}^{2}\right)$ & $40,67(23,3)$ & $37,88(23)$ & $37,47(18,5)$ & 0,018 \\
GC $(\mathrm{l} / \mathrm{min})$ & $5,29(2,9)$ & $4,89(2,9)$ & $4,91(2,2)$ & 0,007 \\
IC $\left(\mathrm{l} / \mathrm{min} / \mathrm{m}^{2}\right)$ & $2,86(1,6)$ & $2,65(1,6)$ & $2,65(1,3)$ & 0,007 \\
\hline
\end{tabular}


Tabla 3 Medias (DT) de los promedios de los indicadores en las tres fases, y niveles críticos ( $p$ ) para las comparaciones por pares, en función de la presencia/ausencia de FA

\begin{tabular}{|c|c|c|c|c|}
\hline \multicolumn{4}{|c|}{ Fase } & \multirow{2}{*}{$\begin{array}{l}P \\
\text { EMP - BiV }\end{array}$} \\
\hline & EMP-anódica & $\mathrm{BiV}$ & VD & \\
\hline \multicolumn{5}{|c|}{ FA no $(n=16)$} \\
\hline $\mathrm{FC}$ & $69,31(10,29)$ & $69,58(9,78)$ & $68,88(9,07)$ & 0,355 \\
\hline PAS & $111,3(13,84)$ & $112,25(11,64)$ & $117,83(19,76)$ & 0,372 \\
\hline PAD & $67,98(12,62)$ & $71,33(11,49)$ & $75,98(16,20)$ & 0,129 \\
\hline PAM & $81,86(15,10)$ & $86,88(11,74)$ & $91,56(17,87)$ & 0,072 \\
\hline VL & $80,73(49,20)$ & $75,26(46,65)$ & $70,7(35,05)$ & 0,034 \\
\hline Ivl & $41,96(24,83)$ & $38,79(23,69)$ & $36,86(17,68)$ & 0,012 \\
\hline GC & $5,48(3,16)$ & $5,1(3,10)$ & $4,81(2,29)$ & 0,008 \\
\hline IC & $2,87(1,62)$ & $2,68(1,60)$ & $2,52(1,20)$ & 0,004 \\
\hline \multicolumn{5}{|c|}{ FA sí $(n=6)$} \\
\hline $\mathrm{FC}$ & $72,45(6,51)$ & $70,80(2,00)$ & $72,03(3,54)$ & 0,208 \\
\hline PAS & $119,55(20,23)$ & $123,25(14,76)$ & $120,82(18,98)$ & 0,201 \\
\hline PAD & $76,93(8,45)$ & $80,78(7,61)$ & $78,82(10,44)$ & 0,066 \\
\hline PAM & $92,03(14,90)$ & $95,98(11,51)$ & $93,72(15,56)$ & 0,092 \\
\hline $\mathrm{VL}$ & $67,17(36,46)$ & $62,62(37,09)$ & $67,48(34,35)$ & 0,150 \\
\hline $\mathrm{iVL}$ & $38,08(21,85)$ & $36,05(23,60)$ & $38,7(21,89)$ & 0,212 \\
\hline GC & $4,92(2,50)$ & $4,48(2,54)$ & $5,1(2,25)$ & 0,149 \\
\hline IC & $2,83(1,60)$ & $2,58(1,61)$ & $2,9(1,45)$ & 0,148 \\
\hline
\end{tabular}

la PAS, PAD y PAM durante las mismas en comparación con la estimulación EMP-anódica (tabla 2). En el análisis por subgrupos se compararon las variables según la presencia de FA (tabla 3) y cardiopatía isquémica (tabla 4). El mayor beneficio se concentró en los pacientes en ritmo sinusal, en quienes se observó un incremento estadísticamente significativo en el iVL $(41,96$ vs. 38,$79 ; p=0,012)$, en el GC $(5,48$ vs. 5,$1 ; p=0,008)$ y en el IC $(2,87$ vs. 2,$68 ; p=0,004)$. Respecto a la presencia de cardiopatía isquémica, se documentó una tendencia a mayor mejoría en la miocardiopatía dilatada no isquémica, aunque dichas diferencias no alcanzaron significación estadística (tabla 4).

Tabla 4 Medias (DT) de los promedios de los indicadores en las tres fases, y niveles críticos (p) para las comparaciones por pares, en función de la presencia/ausencia de CPI

\begin{tabular}{|c|c|c|c|c|}
\hline \multicolumn{4}{|c|}{ Fase } & \multirow{2}{*}{$\begin{array}{l}\mathrm{P} \\
\text { EMP - BiV }\end{array}$} \\
\hline & EMP-Anódica & $\mathrm{BiV}$ & VD & \\
\hline \multicolumn{5}{|c|}{ CPI no $(n=11)$} \\
\hline $\mathrm{FC}$ & $73,91(9,32)$ & $73,36(7,74)$ & $72,45(7,99)$ & 0,339 \\
\hline PAS & $120,21(17,72)$ & $120,23(14,95)$ & $123,70(21,89)$ & 0,498 \\
\hline PAD & $74,22(13,17)$ & $78,58(9,46)$ & $81,19(15,32)$ & 0,047 \\
\hline PAM & $89,10(17,97)$ & $94,52(11,72)$ & $97,55(18,70)$ & 0,064 \\
\hline VL & $69,03(37,91)$ & $62,65(34,66)$ & $66,65(34,71)$ & 0,022 \\
\hline IVl & $38,03(20,52)$ & $35,02(20,24)$ & $37,10(19,83)$ & 0,039 \\
\hline GC & $5,05(2,53)$ & $4,59(2,49)$ & $4,93(2,35)$ & 0,250 \\
\hline IC & $2,83(1,45)$ & $2,56(1,46)$ & $2,75(1,37)$ & 0,022 \\
\hline \multicolumn{5}{|c|}{ CPI sí $(n=7)$} \\
\hline $\mathrm{FC}$ & $64,77(5,61)$ & $64,69(5,09)$ & $65,99(5,64)$ & 0,425 \\
\hline PAS & $104,37(5,94)$ & $109,14(7,12)$ & $111,17(10,55)$ & 0,065 \\
\hline PAD & $65,84(8,11)$ & $68,03(11,02)$ & $70,23(10,04)$ & 0,283 \\
\hline PAM & $79,20(7,98)$ & $89,69(9,45)$ & $84,00(8,75)$ & 0,140 \\
\hline VL & $87,50(55,04)$ & $84,23(53,92)$ & $66,65(34,71)$ & 0,200 \\
\hline Ivl & $44,81(28,35)$ & $42,37(27,87)$ & $38,06(17,84)$ & 0,085 \\
\hline GC & $5,66(3,59)$ & $5,36(3,53)$ & $4,87(2,17)$ & 0,093 \\
\hline IC & $2,91(1,85)$ & $2,77(1,82)$ & $2,49(1,13)$ & 0,097 \\
\hline
\end{tabular}




\section{Discusión}

La captura catódica, que inicia un único frente de activación desde la punta del electrodo de estimulación, es el mecanismo establecido para estimulación del músculo cardiaco. No obstante, algunos estudios en los últimos años han mostrado el potencial beneficio de la captura anódica y la estimulación multipunto en pacientes sometidos a resincronización cardiaca. Entre ellos se ha observado acortamiento en la duración del complejo QRS ${ }^{15,16}$, y mejoría en la función del ventrículo izquierdo medida por la integral bajo la curva del flujo en el tracto de salida del $\mathrm{VI}^{17}$.

Sin embargo, otros estudios, como el de Abu Sham'a et al. ${ }^{18}$, no encontraron diferencias significativas en parámetros hemodinámicos, ecocardiográficos o el efecto global de la resincronización en pacientes con o sin captura anódica, incluso con empeoramiento de la función ventricular izquierda en dos pacientes. Tamborero et al. ${ }^{16}$, a pesar de no ofrecer datos hemodinámicos o ecocardiográficos en los pacientes con captura anódica, postulaban cómo la captura anódica de ventrículo derecho en la configuración punta VI-anillo VD anularía el potencial efecto asociado a la programación del intervalo VV, dado que la activación de ambos ventrículos seguiría siendo simultánea en caso de captura anódica derecha.

La investigación realizada hasta la fecha ha testado el efecto de la estimulación y captura anódica a nivel del ventrículo derecho, cuando la estimulación se realiza entre la punta del electrodo izquierdo y el anillo del electrodo derecho, siendo este efecto de captura anódica derecha mucho mayor en el caso de electrodos bipolares verdaderos ${ }^{19}$ frente a bipolares integrados. Sin embargo, no se ha probado el potencial beneficio de la captura anódica en el ventrículo izquierdo en pacientes portadores de un electrodo cuadripolar implantado en el seno coronario, buscando de esta manera la activación simultánea desde dos puntos diferentes del ventrículo izquierdo, tema que se convirtió en el objetivo de nuestro estudio.

Los resultados obtenidos en este estudio son prometedores en un doble sentido. En primer lugar, se demostró que la estimulación anódica a través de un electrocatéter cuadripolar supuso un incremento significativo, de forma aguda, en el volumen sistólico, el gasto cardiaco y el índice cardiaco en comparación con la estimulación biventricular clásica. Esto presume una clara mejoría en el corazón en su función de bomba y de ello cabe esperar que pudiera implicar una repercusión clínica positiva para estos pacientes en el mediano plazo. No se han observado cambios significativos en la duración del complejo QRS descrito en otros estudios empleando estimulación anódica, si bien es cierto que en ninguno de dichos estudios se había testado un catéter cuadripolar en el seno coronario. La mayor extensión de masa miocárdica capturada en el epicardio, y no en el endocardio donde se encuentran las terminaciones del sistema de Purkinje, podría haber condicionado una duración del QRS no significativamente menor que la encontrada durante la estimulación biventricular convencional. A pesar de ello, la mejoría en los parámetros hemodinámicos agudos fue significativa en los pacientes analizados, con la limitación añadida del bajo tamaño muestral.
Por otro lado, el empleo del Task Force Monitor para la evaluación no invasiva de variables hemodinámicas, supone una ventaja clara de cara a la programación de la mejor estimulación multipunto posible, evitando técnicas más costosas, invasivas y no al alcance de todos los centros implantadores. Recientemente, Pappone et al. ${ }^{20,21}$ mostraron el beneficio hemodinámico agudo de la estimulación multipunto en el VI mediante un catéter cuadripolar, empleando para ello una evaluación invasiva a través de curvas de presión-volumen, y cómo dicho beneficio agudo se traduce en una mejoría de parámetros ecocardiográficos de remodelado y parámetros clínicos en un seguimiento a tres meses. Dado que no existe una única configuración de estimulación multipunto que sea la de mayor beneficio para todos los pacientes, sino que ésta necesita ajustarse por parámetros hemodinámicos, el empleo del Task Force Monitor para la evaluación no invasiva de los mismos supondría una gran ventaja potencial en cuanto a facilidad de empleo, ahorro de costos, ausencia de complicaciones inminentes para el paciente y generabilidad de su aplicación.

De frente a la aplicabilidad clínica de este estudio, quedan aún varias dudas por despejar. En primer lugar, el alto umbral de estimulación requerido para la obtención de captura anódica predeciría una reducción en la vida media de los dispositivos y por tanto un incremento en el número de recambios a los que se sometería el paciente, lo que conllevaría un incremento en la morbilidad y un gasto económico mayor. Hoy en día, la reciente introducción en el mercado de nuevos dispositivos de TRC junto con un nuevo software de programación para electrodo cuadripolar, permite la realización de estimulación multipunto con un consumo de batería muy inferior al que platearía la programación a máximo voltaje para garantizar captura anódica a largo plazo. En la actualidad hay dos estudios aleatorizados en marcha para evaluar la eficacia de la estimulación multipunto en el ventrículo izquierdo mediante un electrodo cuadripolar ${ }^{22}$. El Multipoint Pacing IDE Study (NCT01786993), aleatorizado a implantación de un electrodo cuadripolar vs. bipolar convencional, iniciado en abril de 2013 y con finalización prevista para 2015, y el MOre Response on Cardiac Resynchronization Therapy With MultiPoint Paging (MORE-CRT MPP, NCT02006069), en el que se implanta un electrodo cuadripolar a todos los pacientes, con estimulación bipolar inicial y activación de la estimulación multipunto de forma aleatorizada en los no respondedores a los seis meses, cuyos resultados se esperan para 2017.

En segundo lugar, quedaría por aclarar el potencial efecto proarrítmico de la estimulación anódica del ventrículo izquierdo a través de un electrodo cuadripolar. Bajo algunas circunstancias, el periodo refractario para la estimulación anódica puede ser más corto que para la catódica, como es el caso de extraestímulos muy próximos y durante el periodo refractario relativo. Esto probablemente se debe a que los impulsos hiperpolarizantes incrementan la disponibilidad del canal de sodio y por tanto aceleran la despolarización. También se ha descrito una prolongación en el intervalo $\mathrm{QT}^{23}$ con estimulación anódica. Si bien, en ambos casos se desconoce el resultado que tendría dicha estimulación anódica cuando se realiza en el ventrículo izquierdo, máxime considerando sus potenciales beneficios en la respuesta hemodinámica del paciente, finalmente podría traducirse en una reducción de episodios arrítmicos 
en el mediano y largo plazo en pacientes respondedores clínicos y ecocardiográficos, en consonancia con la literatura publicada. En tercer lugar, habría que valorar cómo la mejora de estos parámetros agudos se convierte en una mejoría de parámetros clínicos, como la clase funcional, la calidad de vida o la mortalidad, así como de parámetros ecocardiográficos de remodelado, en el mediano y largo plazo.

\section{Limitaciones}

Las limitaciones son inherentes a todo estudio observacional, no aleatorizado, monocéntrico, con escaso número de pacientes. Sin embargo, el hecho de que cada paciente haya actuado como su propio control permitió aumentar la potencia estadística y llegar a documentar diferencias estadísticamente significativas de forma aguda a pesar del escaso tamaño muestral. Será necesario el desarrollo de estudios prospectivos y aleatorizados con mayor número de pacientes y objetivos clínicos y de remodelado a mediano y largo plazo, que ayuden a despejar las dudas que quedan sin resolver y previamente fueron mencionadas.

El hecho de haber analizado el beneficio de la captura multipunto anódica, cuando ya existe disponibilidad de dispositivos que permiten captura multipunto catódica, hace que dichos resultados hayan de ser replicados con los nuevos modos de estimulación. En cualquier caso, no parece probable que, dado el beneficio agudo documentado, dicho resultado no sea igualmente evidenciable con estimulación catódica multipunto.

\section{Conclusiones}

La estimulación anódica-EMP mediante un electrodo cuadripolar en el VI es factible. Incluso en una pequeña población de pacientes se demostraron diferencias significativas en el volumen latido y gasto cardiaco, limitado a la población en ritmo sinusal. Si bien el pequeño tamaño de la muestra, limita la demostración de diferencias significativas entre grupos, el beneficio sugiere ser mayor en la miocardiopatía dilatada no isquémica. El empleo del Task Force Monitor en la evaluación hemodinámica aguda de estos pacientes, facilitaría la programación adecuada de la estimulación multipunto y la mayor difusión de la terapia en condiciones óptimas.

\section{Responsabilidades éticas}

Protección de personas y animales. Los autores declaran que los procedimientos seguidos se conformaron a las normas éticas del comité de experimentación humana responsable y de acuerdo con la Asociación Médica Mundial y la Declaración de Helsinki.

Confidencialidad de los datos. Los autores declaran que han seguido los protocolos de su centro de trabajo sobre la publicación de datos de pacientes.

Derecho a la privacidad y consentimiento informado. Los autores han obtenido el consentimiento informado de los pacientes y/o sujetos referidos en el artículo. Este documento obra en poder del autor de correspondencia.

\section{Financiación}

Ninguna.

\section{Conflictos de intereses}

- Dr Toquero: honorarios de consultoría para Boston Scientific, Medtronic y St Jude Medical. Asistencia, participación y honorarios en simposium patrocinados

- Dr. Sánchez: participación en ensayos clínicos patrocinados por St Jude Medical

- Dra. Ruíz: participación en ensayos clínicos patrocinados por St Jude Medical

- Dr. Castro: participación en ensayos clínicos patrocinados por St Jude Medical

- Sra. Sara Alyoun: Ninguno

- Dr. Fernández Lozano: honorarios de consultoría para Boston Scientific y St Jude Medical. Asistencia, participación y honorarios en simposium patrocinados

\section{Bibliografía}

1. Abraham WT, Fisher WG, Smith AL, Delurgio DB, Leon AR, Loh $\mathrm{E}$, et al. Cardiac resynchronization in chronic heart failure. N Engl J Med. 2002;346:1845-53.

2. Young JB, Abraham WT, Smith AL, Leon AR, Lieberman R, Wilkoff $B$, et al. Combined cardiac resynchronization and implantable cardioversion defibrillation in advanced chronic heart failure: The MIRACLE ICD Trial. JAMA. 2003;289:2685-94.

3. Cleland JG, Daubert JC, Erdmann E, Freemantle N, Gras $D$, Kappenberger $L$, et al. Longer-term effects of cardiac resynchronization therapy on mortality in heart failure [the CArdiac REsynchronization-Heart Failure (CARE-HF) trial extension phase]. Eur Heart J. 2006;27:1928-32.

4. Bradley DJ, Bradley EA, Baughman KL, Berger RD, Calkins H, Goodman SN, et al. Cardiac resynchronization and death from progressive heart failure: A meta-analysis of randomized controlled trials. JAMA. 2003;289:730-40.

5. Diaz-Infante E, Mont L, Leal J, Garcia-Bolao I, Fernandez-Lozano I, Hernandez-Madrid A, et al. Predictors of lack of response to resynchronization therapy. Am J Cardiol. 2005;95:1436-40.

6. Stockburger M, Fateh-Moghadam S, Nitardy A, Langreck H, Haverkamp W, Dietz R. Optimization of cardiac resynchronization guided by Doppler echocardiography: Haemodynamic improvement and intraindividual variability with different pacing configurations and atrioventricular delays. Europace. 2006;8:881-6.

7. Bertini M, Delgado V, Bax JJ, Van de Veire NR. Why, how and when do we need to optimize the setting of cardiac resynchronization therapy? Europace. 2009; 11(Suppl. 5):v46-57.

8. Gras D, Gupta MS, Boulogne E, Guzzo L, Abraham WT. Optimization of $A V$ and VV delays in the real-world CRT patient population: An international survey on current clinical practice. Pacing Clin Electrophysiol. 2009;32 Suppl. 1:S236-9.

9. Khan FZ, Virdee MS, Pugh PJ, Read PA, Fynn SP, Dutka DP. Noninvasive cardiac output measurements based on bioreactance for optimization of atrio- and interventricular delays. Europace. 2009;11:1666-74.

10. Squara P, Denjean D, Estagnasie P, Brusset A, Dib JC, Dubois C. Noninvasive cardiac output monitoring (NICOM): A clinical validation. Intensive Care Med. 2007;33:1191-4. 
11. Lang CC, Karlin P, Haythe J, Lim TK, Mancini DM. Peak cardiac power output, measured noninvasively, is a powerful predictor of outcome in chronic heart failure. Circ Heart Fail. 2009;2:33-8.

12. Khan FZ, Virdee MS, Hutchinson J, Smith B, Pugh PJ, Read PA, et al. Cardiac resynchronization therapy optimization using noninvasive cardiac output measurement. Pace. 2011;34:1527-36.

13. Irwin ME, Thangaroopan M, Gulamhusein SS. Electrocardiography of cardiac resynchronization therapy: Phenomenon of left cathode and rigth anodal capture. (abstract 882). Heart Rhythm. 2004; 1(1):S276.

14. Mounsey JP, Knisley SB, Anodal Capture. Cathodal Capture, and Left Ventricular Cardiac Excitation. J Cardiovasc Electrophysiol. 2009;20:650-2.

15. Tedrow UB, Stevenson WG, Wood MA, Shepard RK, Hall K, Pellegrini $\mathrm{CP}$, et al. Activation sequence modification during cardiac resynchronization by manipulation of left ventricular epicardial pacing stimulus strength. PACE. 2007;30:65-9.

16. Tamborero D, Mont L, Alanis R, Berruezo A, Tolosana JM, Sitges $M$, et al. Anodal capture in cardiac resynchronization therapy implications for device programming. PACE. 2006;29:940-5.

17. Lloyd M, Heeke S, Lerakis S, Langberg JJ. Reverse polarity pacing: the hemodynamic benefit of anodal currents at lead tips for cardiac resynchronization therapy. J Cardiovasc Electrophysiol. 2007;18:1167-71.
18. Abu Sham'a R, Kuperstein R, Barsheshet A, Bar-Lev D, Luria $D$, Gurevitz $O$, et al. The effects of anodal stimulation on electrocardiogram, left ventricular dyssynchrony, and acute haemodynamics in patients with biventricular pacemakers. Europace. 2011;13(7):997-1003.

19. Freedman RA, Petrakian A, Boyce K, Haffajee C, Val-Mejías J, Oza AL. Performance of dedicated versus integrated bipolar defibrillator leads with CRT-defibrillators: results from a prospective multicenter study. PACE. 2009;32:157-65.

20. Pappone C, Ćalović Ž, Vicedomini G, Cuko A, McSpadden LC, Ryu K, et al. Multipoint left ventricular pacing improves acute hemodynamic response assessed with pressure-volume loops in cardiac resynchronization therapy patients. Heart Rhythm. 2014;11(3):394-401

21. Pappone C, Ćalović Ž, Vicedomini G, Cuko A, McSpadden LC, Ryu $\mathrm{K}$, et al. Multipoint left ventricular pacing in a single coronary sinus branch improves mid-term echocardiographic and clinical response to cardiac resynchronization therapy. J Cardiovasc Electrophysiol. 2015;26(1):58-63.

22. Rinaldi CA, Burri H, Thibault B, Curnis A, Rao A, Gras D, et al. A review of multisite pacing to achieve cardiac resynchronization therapy. Europace. 2015;17:7-17.

23. Mounsey JP, Knisley SB. Anodal capture, cathodal capture, and left ventricular cardiac excitation. J Cardiovasc Electrophysiol. 2009;20:650-2. 